\title{
Validation of the Personal Wellbeing Index for People with End Stage Kidney Disease
}

\author{
Melissa K. Weinberg ${ }^{1}$ • Paul N. Bennett ${ }^{1,2}$. \\ Robert A. Cummins ${ }^{1}$
}

Received: 11 January 2015 / Accepted: 7 September 2015 / Published online: 15 September 2015 (C) Springer Science+Business Media Dordrecht and The International Society for Quality-of-Life Studies (ISQOLS) 2015

\begin{abstract}
The quality of life of people with end stage kidney disease (ESKD) has traditionally been measured using instruments that emphasise objective health status. The present study validates an alternative measure, the Personal Wellbeing Index (PWI), which measures subjective wellbeing. An Australian ESKD sample $(N=172$, Mean age $=64.04, S D=14.82$ ) completed the PWI as well as health-specific quality of life measures. The PWI was subject to confirmatory factor analysis, and a series of regressions and between-group comparisons were performed to reveal that it is a psychometrically appropriate measure for this sample. The PWI and health-specific measures each yield different and complementary results. Thus, the PWI is proposed as a complement to existing health-related quality of life tools, in order to broaden understanding of the patient's subjective experience. The resulting profile is argued to better inform targeted interventions to improve the quality of life of people with ESKD.
\end{abstract}

Keywords Subjective wellbeing · Quality of life · End-stage kidney disease

The quality of life of people with end stage kidney disease (ESKD) has been commonly assessed using both generic health-related and specific disease-related tools (e.g., Abdel-Kader et al. 2009b; Ekelund and Andersson 2007; Al-Arabi 2006; Ibrahim and El Salamony 2008; Chan et al. 2009). However, contemporary approaches to wellbeing from within the social-sciences recognize that health is just one aspect of life that contributes to overall life satisfaction (Cummins 2010a). This is true even for very serious medical conditions, such as ESKD, defined as the final stage of chronic

Melissa K. Weinberg

melissaw@ deakin.edu.au

1 Faculty of Health, Deakin University, Melbourne, Australia

2 Western Health Nursing Research Centre, Melbourne, Australia 
kidney disease where the glomerular filtration rate falls below $15 \mathrm{~mL} / \mathrm{min}$ (Sinclair et al. 2014). It is at this stage that the person must choose to undergo dialysis, kidney transplantation, supportive therapy, or to withdraw from active treatment. These are clearly major decisions and an understanding of how such people are perceiving them self and their lives is crucial to offering professional advice.

Although living with ESKD poses an undeniable challenge it is also understood that people generally adapt to their objective life circumstances, as the impact of their health condition becomes integrated into their lifestyle (Suh et al. 1996; Diener et al. 1999; Okun and George 1984). Thus, measuring wellbeing solely through health likely overemphasizes the impact that a patient's objective health status has on their own perception of their overall life quality. In addition, wellbeing measurement restricted to healthspecific scales does not allow wellbeing comparisons with the general population. For these reasons, this study examines a complementary indicator in the form of subjective wellbeing.

The use of health-specific measures to evaluate patient quality of life follows the traditional medical model, wherein complete health is defined as the absence of disease or illness. This is evident in questionnaires such as the Kidney Disease Quality of Life scale (Hays et al. 1994) scale and the Dialysis Symptom Index (Weisbord et al. 2004), which were designed specifically to assess the health-related quality of life of people with ESKD on dialysis. These scales assume that quality of life improves in a linear fashion with symptom relief. However, such scales do not fully match best-practice health care, which promotes a 'person-centred' approach (Epstein et al. 2010) extending beyond health-related quality of life. These scales also do not fulfil the most recent definition of health adopted by the World Health Organization (WHO 2012).

In 1948, WHO defined health as "a state of complete physical, mental and social well-being and not merely the absence of disease or infirmity". Clearly, therefore, even this old definition challenges the use of scales that associate health or quality of life with disease-specific symptoms. In 2013, WHO (Europe) endorsed an even more far reaching definition as "Well-being exists in two dimensions, subjective and objective. It comprises an individual's experience of their life as well as a comparison of life circumstances with social norms and values" (WHO 2013: p.4-5). In accordance with this definition, psychologists and quality of life researchers are increasingly turning to Subjective Wellbeing (SWB) as an indicator of quality of life within the WHO definition.

Certainly within the ESKD literature, some studies have considered psychological wellbeing beyond health-related quality of life. However, such measures have typically emphasised adverse psychological states such as depression (Abdel-Kader et al. 2009a), or fatigue (Bonner et al. 2013). For example, a recent study of people receiving haemodialysis in Scotland (Alshraifeen et al. 2014) claimed to measure psychological wellbeing using four subscales: somatic symptoms, anxiety and insomnia, social dysfunction and severe depression. Crucially, however, the relationship between illbeing and wellbeing is not linear (Cummins 2010a). Because of this non-linearity, finding that the ESKD population report higher scores on these illbeing measures cannot be used to infer that they are lower in terms of psychological wellbeing.

A further consideration for understanding is that correlations between measures of negative emotional states and health-related quality of life may be inflated due to overlap of symptoms. For example, measures like the Depression Anxiety and Stress 
Scales (DASS; Lovibond and Lovibond 1995) include items that are both symptoms reflecting these psychological states, as well as recognised side-effects of ESKD. For example, the anxiety subscale includes items regarding physiological conditions, like "I was aware of dryness in my mouth", which is extremely common among people receiving hemodialysis. Thus, these measures confound the physiological consequences of the condition with the psychological state being measured. Evaluating quality of life using a measure of subjective wellbeing, then, arguably provides a more accurate representation of a patient's general psychological state, without the associated redundancy that conflates associations between variables.

Further, measuring subjective wellbeing allows the adoption of a framework within which to better understand the complex processes that help to manage and maintain psychological wellbeing in times of challenge. Specifically, the homeostasis theory of subjective wellbeing (Cummins et al. 2003; Cummins 2010b) describes how internal psychological mechanisms work to regulate emotions around a genetically predetermined set-point for mood happiness. According to theory, when life events are encountered that threaten wellbeing, a system of psychological resources is engaged to alleviate the negative impact of the event on wellbeing and effectively exert resilience.

Because homeostasis confers resilience, subjective wellbeing has a complex relationship with all of the traditional objective measures of wellbeing like income, life expectancy, or health status (Cummins 2010a; Okun and George 1984; Diener et al. 1999). This relationship is a function of the interactive homeostatic processes that seek to maintain SWB within a normal range (Cummins and Wooden 2013; Cummins 2010b). This function is effective in explaining the non-linear relationship between SWB and objective measures of health.

SWB can be further deconstructed into a set of domain-specific measures, which comprise the areas of life most predictive of general life satisfaction. The scale that best represents this form of measurement, and the one recommended for this purpose by WHO (WHO 2013) and the Organization for Economic Co-operation and Development (OECD 2013) is the Personal Wellbeing Index (PWI; The International Wellbeing Group 2006). Importantly in the current context, the PWI has been validated in a sample of adults living with a chronic medical condition in the form of HIV (Hutton et al. 2013), demonstrating adequate psychometric performance in relation to health constructs. These authors also recommend the PWI as an appropriate tool to evaluate the SWB of people with other chronic health conditions.

The PWI is purposefully designed with the intent to deconstruct General Life Satisfaction (GLS) into its explanatory components. GLS is measured by a single question concerning satisfaction with 'life as a whole', and the PWI measures SWB through satisfaction with 7 domains as: standard of living, health, achieving in life, relationships, safety, community, and future security (The International Wellbeing Group 2006). The scale has a deliberate construction whereby each domain earns inclusion by making a significant unique statistical contribution to GLS. It is notable that in surveys of the general Australian population, the unique contribution of health satisfaction pales in comparison to the contribution made by other domains (Cummins et al. 2013). This also holds for samples in which people have specific health concerns.

For example, Lau et al. (2008) found that for people who were indirectly exposed to Severe Acute Respiratory Syndrome in Hong Kong, the domain contribution by standard of living and achieving in life exceeded the contribution of the health domain. 
Such results confirm the remarkable ability of people to adapt to circumstances of chronic threat to their physical health.

The current study aims to validate the PWI for use in a sample of people with endstage kidney disease. This validation will proceed through a confirmatory factor analysis, followed by a comparison of subjective wellbeing indicators and diseasespecific measures to account for overall life satisfaction.

\section{Method}

\section{Participants}

A sample of 172 participants who were receiving haemodialysis to manage end-stage kidney disease (ESKD) was recruited from a sampling frame of 385 patients from 8 separate dialysis centres in the Eastern and Southern suburbs of Melbourne. The study was advertised on flyers posted in the dialysis centres, and a research assistant attended the units to explain the nature of the study and administer the questionnaires to consenting participants. The sample included 104 males $(60.5 \%)$ and $66(38.4 \%)$ females. Their average age was $64.04(\mathrm{SD}=14.82)$. The majority $(61 \%)$ was married, and about $80 \%$ were not engaged in paid work, reporting a household income of less than $\$ 60,000$ per year. Participants spent an average of $13.45 \mathrm{~h}$ per week on dialysis.

A comparative sample of 200 participants, of similar age, was systematically extracted from the $23^{\text {rd }}$ longitudinal survey of the Australian Unity Wellbeing Index conducted in early 2012 (Cummins and Weinberg 2012). The raw data from this survey are available from the first author. The extracted sample is of comparative age $(\mathrm{M}=$ 63.97, $\mathrm{SD}=14.68$ ) and the survey includes critical variables in common with the questionnaire for the ESKD patients. The full sample was reduced to provide a comparison group of random Australian participants with a similar age distribution to the ESKD group. The general Australian sample contains a more even spread of males $(50.5 \%)$ to females $(49.5 \%)$, but were similar with regard to their marital status $(60 \%$ married) with $63 \%$ not engaged in paid work. Since there is no evidence of gender differences for the wellbeing variables in the original file from which this sample was extracted, the sample is considered to be an appropriate comparison group.

\section{Measures}

Participants completed a questionnaire designed to measure various aspects of their wellbeing, including their subjective wellbeing, mood, self-esteem, optimism, and perceived control. Scales to measure quality of life specifically in relation to participants' kidney disease were also included.

Subjective Wellbeing (SWB) was measured in two ways. The first, general life satisfaction (GLS), was measured using the single item "How satisfied are you with your life as a whole?" Participants rated their level of satisfaction on an 11-point, enddefined scale anchored by "Not satisfied at all" to "Completely satisfied". To gauge a more domain-oriented assessment of SWB, the seven-item Personal Wellbeing Index (The International Wellbeing Group 2006) was included, whereby participants rate their 
satisfaction with seven domains of life as: standard of living, health, achieving in life in life, relationships, safety, community, and future security. Respondents use the same 11-point scale as for GLS. The scale has strong psychometric properties including adequate test-retest reliability with Cronbach's alpha ranging between 0.70 and 0.85 (The International Wellbeing Group 2006). Convergent validity has also been established via correlation with the Satisfaction with Life Scale $(r=0.78)$ (Diener et al. 1985). In the present study, Cronbach's alpha reliability scores for the ESKD sample and the general Australian sample were both 0.89 .

Health Related Quality of Life (HRQOL) Selected items from the Dialysis Symptom Index (DSI) (Weisbord et al. 2004) were chosen based on the rates that they were endorsed by ESKD patients in multiple studies (Ibrahim and El Salamony 2008; Abdel-Kader et al. 2009a, b; Son et al. 2009). In these studies, participants rated the frequency with which they experienced each symptom, and responses across the four studies were ranked and compared to determine the most common symptoms. In the present study, participants rated how much they felt bothered by each symptom on a scale from 0 ("not at all") to 10 ("extremely"). These 12 items were light-headedness, muscle cramps, numbness, feeling tired, dry mouth, bone or joint pain, dry skin, itching, trouble falling asleep, trouble staying asleep, and problems with your access site. These factors are important to establishing the overarching effect of kidney disease, and treatment burden of the person with ESKD (Karamanidou et al. 2014). The decision to use a shortened version of the DSI was governed by a desire to minimise overall questionnaire length to reduce the opportunity for respondent fatigue, along with recommendations from the original scale authors that the 30 item version is intended as a preliminary instrument only. Scores on the 12 items were averaged to gauge an amount of bother due to these symptoms. Reliability for this scale was high in the present study, with a Cronbach's alpha of 0.85 .

In addition, the KD-QOL (short form) (Hays et al. 1994) was also included. Of the 11 subscales, seven were included in the present study, as: Effects of kidney disease, the burden of kidney disease, patients' cognitive and social functioning, the social support they receive, and their satisfaction with their dialysis staff and with their patient experience. Items include "I had difficulty concentrating or thinking" and "Dialysis staff encourage me to be as independent as possible", and respondents rated each on a 0-10 scale. Four subscales from the KD-QOL were not included because they overlapped with other measures (e.g., the Symptoms subscale is essentially a shortened measure of the DSI, as described above). Because the subscales are scored independently, the removal of these four subscales does not affect the interpretation of the subscales retained. Cronbach's alpha was acceptable for all subscales, ranging between 0.76 (social support) and 0.90 (burden of kidney disease).

Self Esteem The five positively-worded items from the Rosenberg Self Esteem Scale (Rosenberg 1965) were used to evaluate self-esteem. Although the original RSES scale comprises 10 items, factor analyses conducted on the full scale results in the emergence of two factors, reflecting methods effects (Owens 1994; Sheasby et al. 2000; Tafarodi and Swann 1995; Marsh 1996; Corwyn 2000; Martin et al. 2006; Roth et al. 2008). When such factors emerge, the factor comprising negative items reflects a tendency 
towards self-deprecation (Owens 1994), which is not consistent with Rosenberg's original definition of self-esteem. Thus, using only positive items produces a single factor which is both a more parsimonious and accurate representation of the construct. Participants rated how much they agree with statements such as "I feel I have a number of good qualities" on an 11-point scale ranging from 0 "Do not agree at all" to 10 "Agree completely". In the present study, Cronbach's alpha reliability scores were high for both samples, with Cronbach's alpha at 0.91 for the general Australian sample, and 0.88 for the ESKD group.

Perceived Control The first five items from Pearlin and Schooler's (1978) Mastery subscale were used to assess perceived control. These items represent a sense of general control over one's life and the capacity to effect change, and include "I have little control over the things that happen to me". These items were rated on a $0-10$ scale as above. The final two items of the scale were not included because they are worded in the opposite direction to the first five items and display the weakest factor loadings (Pearlin and Schooler 1978). Given that high scores on this scale indicate low perceived control, scores were reversed so that high scores reflect high levels of control. For the present study, Cronbach's alpha for the scale was 0.88 for the general Australian sample, and 0.83 for the ESKD group.

\section{Procedure}

Ethical approval was obtained from Human Research Ethics Committees of Southern Health \#12143B, Eastern Health \#LR32/1213 and Deakin University \#2012-271. Participants voluntarily returned participant consent forms during the following dialysis session, thereby allowing them to discuss their participation with significant others before providing written informed consent. Both the ESKD sample and the comparison group completed hard-copies of the questionnaire and returned them to the researchers in sealed envelopes.

\section{Data Cleaning and Preparation}

Data were entered into SPSS Version 21.0 and checked for missing values. Prior to data cleaning, all scores for continuously measured variables were transformed from a $0-10$ scale to a standard 0-100 scale. Aggregate scores were computed for each scale and the resulting variables were screened for outliers. A total of 13 univariate outliers were identified in the dataset as being three standard deviations beyond the mean. These scores were recoded back to within the accepted range as recommended by Pallant (2007). The data were then screened for multivariate outliers using Mahalanobis distance. No cases exceeded the critical $\chi^{2}$ value of $46.80(d f=21, p<.001)$. Finally, four cases were removed from the dataset because their responses were suggestive of an acquiescent response style, following recommendation from the PWI user-manual (The International Wellbeing Group 2006). Following these procedures, a confirmatory factor analysis of the PWI was conducted for the ESKD sample, and the descriptive statistics, together with correlations, regressions, and between-group comparisons are presented. 


\section{Results}

To check that the PWI loaded onto a single factor for the ESKD sample, a confirmatory factor analysis was conducted. The analysis revealed that a single factor solution fit the data. Although the overall chi-square value was statistically significant $\left(x^{2}=38.487\right.$, $p<0.001$ ), it is alone insufficient to determine adequate model fit, and other fit indices, including CFI (0.95), TLI (0.93), and SRMR (0.0453) attested to the appropriateness of the model. In addition, the proportion of chi-square to degrees of freedom was minimal $\left(x^{2} / d f=2.749\right)$. Although the RMSEA value $(0.107)$ was slightly above the recommended value for good fit by $\mathrm{Hu}$ and Bentler (1999), modification indices suggested that allowing some error terms to co-vary would only marginally improve the model, and so the model was retained in its tested form. The model, with standardised factor loadings, is shown in Fig. 1.

Figure 1 reveals that the domain of future security loaded highest onto the latent factor, followed by safety and standard of living. Overall, the factor structure of the PWI is acceptable in the ESKD sample.

For comparative purposes and to provide context, means, standard deviations, and correlations among measured variables for both samples are shown in Table 1.

Whereas for the general sample, scores for GLS and SWB lie well within their respective normal ranges (76.01 to 79.17 , and 73.83 to 76.71 points, respectively) (Cummins et al. 2013), the PWI value for the ESKD sample lies 10 points below the score for the general Australian sample. Further investigation of the PWI domains indicated that this difference was largely driven by differences in two key domains: satisfaction with health (ESKD sample: $M=47.67, S D=24.36$; General sample: $M=$ $70.15, S D=20.21$ ) and satisfaction with achieving in life (ESKD sample: $M=54.34$, $S D=26.43$; General sample: $M=70.96, S D=21.66$ ). These differences have already been reported in a previous paper (Bennett et al. 2015) but are reproduced here to provide context.

Table 1 also reveals that for the ESKD sample, the PWI was most strongly correlated with general life satisfaction, as has been repeatedly demonstrated in general Australian samples. It is interesting to note that Control had only weak correlations with other

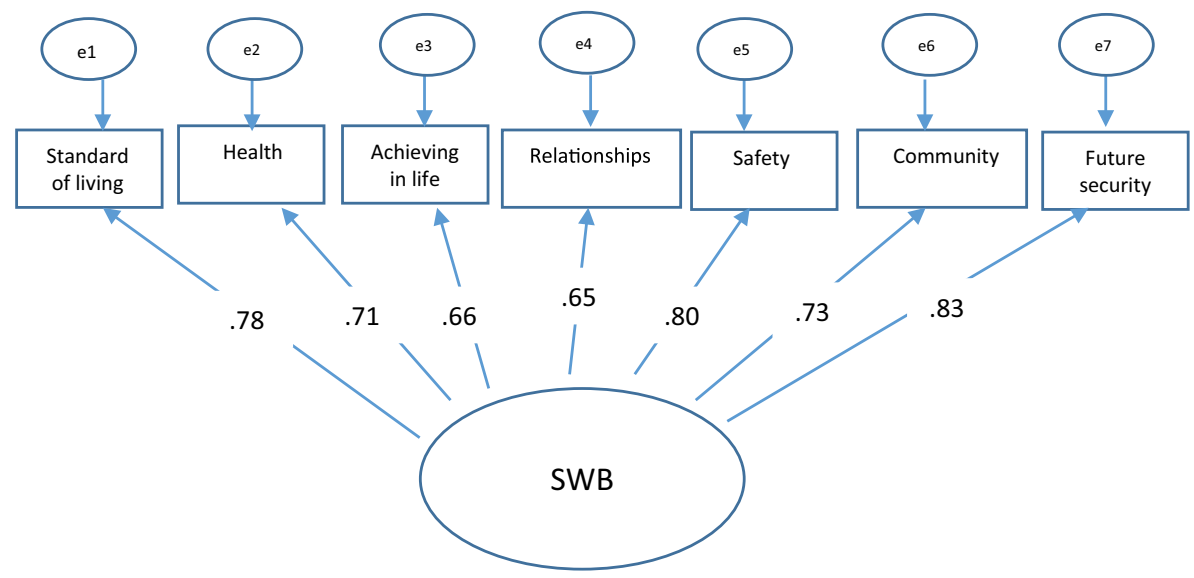

Fig. 1 Confirmatory factor analysis of the PWI 


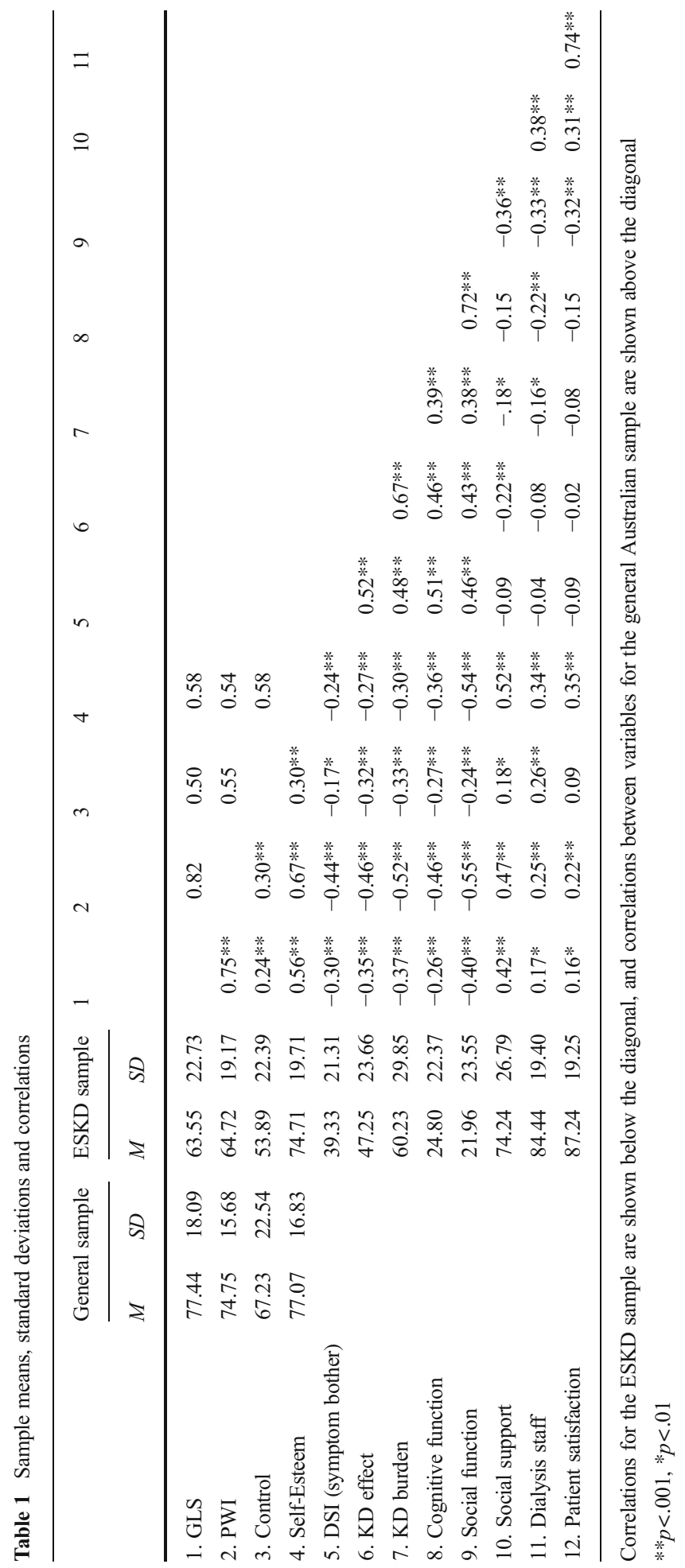


psychological wellbeing measures in the ESKD sample, though correlations were as expected in the general Australian sample. For people with ESKD, patient satisfaction was most strongly linked to satisfaction with dialysis staff, and the subjective wellbeing measures were most strongly correlated with the social domains of the KD-QOL. These theoretically consistent relationships support the construct validity of the PWI in these samples.

For the common measures, t-tests revealed significant differences between the ESKD sample and the general Australian sample on GLS, PWI, and control. However, there was no significant difference in self-esteem between the groups.

What is most evident from these values is the high degree of variation within each measure for the ESKD sample. This likely reflects high variability in the extent to which individuals experience the symptoms and consequences of their disease.

In order to test whether the disease-specific scales adequately capture patients' general subjective quality of life, general life satisfaction was regressed on the subscales of the KD-QOL and DSI. This analysis was conducted as part of a hierarchical multiple regression, which enabled the other psychological variables to be entered at Steps 2 and 3. The results are shown in Table 2.

Together, the KD-QOL and DSI sub-scales predicted only $31.3 \%$ of the variance in GLS when entered into the model at Step 1. Social support $(\beta=0.320)$ was the only significant unique contributor, accounting for about $8 \%$ of variance alone. This finding supports the relatively poor ability of disease-specific measures to represent subjective states. When Self Esteem and Control were entered into the model at Step 2, social support remained a statistically significant unique contributor, but its capacity to predict GLS was overwhelmed by the inclusion of self-esteem. The addition of these psychological variables significantly improved the predictive capacity of GLS to $40.5 \%$ (Fchange [2127]=9.760, $p<.001$ ). At the final step of the model, the total PWI score was added, and all other predictors became non-significant. The PWI alone accounted for $18 \%$ of variance in overall GLS, and raised the total predictive capacity of the model to $58.9 \%$ (Fchange [1126]=56.606, $p<.001$. When the PWI was included, it dominated the prediction of GLS, rendering other indicators largely ineffective.

To further determine the utility of the DSI and KD-QOL subscales to predict subjective states, the domain of health satisfaction from the PWI scale was regressed onto the KD-QOL and DSI scales. This was performed because, although they were unable to significantly predict general life satisfaction in the context of other psychological variables, they may still provide valuable insight into the health-specific domain. The results are shown in Table 3.

In this analysis, the KD-QOL and DSI subscales predicted $37 \%$ of the variance in health satisfaction, with the burden of disease $(\beta=-0.327)$ the strongest unique predictor. The bother experienced by symptoms as measured by the DSI was also a significant unique predictor $(\beta=-0.236)$ as was the level of social support received $(\beta=0.182)$.

\section{Discussion}

The aim of this study was twofold. First to explore the quality of life of people with end-stage kidney disease (ESKD) beyond their health status, using measures of subjective wellbeing (SWB). Second, to compare the relationship between SWB and two scales measuring health-related quality of life (HRQOL). 
Table 2 Hierarchical multiple regression of general life satisfaction on HR-QoL measures and psychological variables

\begin{tabular}{|c|c|c|c|c|c|c|}
\hline \multirow{2}{*}{ Predictor } & & \multicolumn{5}{|c|}{$\mathrm{DV}=\mathrm{General}$ life satisfaction } \\
\hline & & $\mathrm{r}^{\mathrm{a}}$ & Beta & $\mathrm{t}$ & $\mathrm{sr}^{2 \mathrm{~b}}$ & $\mathrm{p}$ \\
\hline \multirow[t]{8}{*}{ Step 1} & DSI & -0.30 & -0.09 & -0.95 & 0.00 & 0.345 \\
\hline & Effect & -0.35 & -0.06 & -0.57 & 0.00 & 0.570 \\
\hline & Burden & -0.37 & -0.19 & -1.87 & 0.02 & 0.064 \\
\hline & Cognitive function & -0.26 & 0.09 & 0.78 & 0.00 & 0.440 \\
\hline & Social function & -0.40 & -0.22 & -1.87 & 0.02 & 0.063 \\
\hline & Social support & 0.42 & 0.32 & 3.79 & 0.08 & 0.000 \\
\hline & Dialysis staff & 0.17 & -0.07 & -0.62 & 0.00 & 0.536 \\
\hline & Patient satisfaction & 0.16 & 0.03 & 0.27 & 0.00 & 0.786 \\
\hline \multirow[t]{10}{*}{ Step 2} & DSI & -0.30 & -0.10 & -1.12 & 0.01 & 0.265 \\
\hline & Effect & -0.35 & -0.07 & -0.71 & 0.00 & 0.482 \\
\hline & Burden & -0.37 & -0.13 & -1.36 & 0.01 & 0.177 \\
\hline & Cognitive function & -0.26 & 0.11 & 1.01 & 0.00 & 0.317 \\
\hline & Social function & -0.40 & -0.10 & -0.83 & 0.00 & 0.406 \\
\hline & Social support & 0.42 & 0.18 & 2.09 & 0.02 & 0.039 \\
\hline & Dialysis staff & 0.17 & -0.06 & -0.53 & 0.00 & 0.599 \\
\hline & Patient satisfaction & 0.16 & -0.03 & -0.28 & 0.00 & 0.778 \\
\hline & Self-Esteem & 0.56 & 0.40 & 4.26 & 0.09 & 0.000 \\
\hline & Control & -0.24 & -0.03 & -0.39 & 0.00 & 0.696 \\
\hline \multirow[t]{11}{*}{ Step 3} & DSI & -0.30 & 0.03 & 0.04 & 0.00 & 0.968 \\
\hline & Effect & -0.35 & -0.07 & -0.84 & 0.00 & 0.404 \\
\hline & Burden & -0.37 & 0.04 & 0.47 & 0.00 & 0.641 \\
\hline & Cognitive function & -0.26 & 0.15 & 1.70 & 0.01 & 0.092 \\
\hline & Social function & -0.40 & -0.05 & -0.54 & 0.00 & 0.589 \\
\hline & Social support & 0.42 & 0.06 & 0.81 & 0.00 & 0.419 \\
\hline & Dialysis staff & 0.17 & -0.04 & -0.40 & 0.00 & 0.692 \\
\hline & Patient satisfaction & 0.16 & -0.02 & -0.16 & 0.00 & 0.872 \\
\hline & Self-Esteem & 0.56 & 0.10 & 1.11 & 0.00 & 0.267 \\
\hline & Control & -0.24 & -0.02 & -0.30 & 0.00 & 0.763 \\
\hline & PWI & 0.75 & 0.70 & 7.52 & 0.18 & 0.000 \\
\hline
\end{tabular}

${ }^{\text {a }}$ zero-order correlation between each predictor and the DV

$\mathrm{b}$ the proportion of unique variance in the DV explained by each predictor

c Significant unique predictors $(p<.05)$ are highlighted in bold

The use of the PWI as an indicator of subjective wellbeing (SWB) in this sample was supported psychometrically. The reliability of the instrument for the ESKD sample was equivalent to the general Australian population, and the items formed a single factor, as expected. Further, the correlations between the PWI and other wellbeing measures (except control) were similar for both groups, supporting construct validity. 
Table 3 Standard multiple regression of health satisfaction on DSI and KD-QOL subscales

\begin{tabular}{|c|c|c|c|c|c|}
\hline \multirow[b]{2}{*}{ Predictor } & \multicolumn{5}{|c|}{ DV = Satisfaction with health } \\
\hline & $\mathrm{r}^{\mathrm{a}}$ & Beta & $\mathrm{t}$ & $\mathrm{sr}^{2 \mathrm{~b}}$ & $\mathrm{p}$ \\
\hline DSI & -0.44 & -0.24 & -2.592 & 0.03 & 0.011 \\
\hline Effect & -0.44 & -0.08 & -0.774 & 0.00 & 0.441 \\
\hline Burden & -0.51 & -0.33 & -3.359 & 0.05 & 0.001 \\
\hline Cognitive function & -0.25 & 0.12 & 1.087 & 0.01 & 0.279 \\
\hline Social function & -0.34 & -0.08 & -0.688 & 0.00 & 0.493 \\
\hline Social support & 0.30 & 0.18 & 2.262 & 0.02 & 0.025 \\
\hline Dialysis staff & 0.16 & -0.10 & -0.867 & 0.00 & 0.388 \\
\hline Patient satisfaction & 0.21 & 0.17 & 1.534 & 0.01 & 0.128 \\
\hline
\end{tabular}

${ }^{\text {a }}$ zero-order correlation between each predictor and the DV

$\mathrm{b}$ the proportion of unique variance in the DV explained by each predictor

c Significant unique predictors $(p<.05)$ are highlighted in bold

Thus, the PWI appears to be a psychometrically appropriate measure of subjective wellbeing for the ESKD population.

This finding is especially interesting since, also as expected, the ESKD sample reported substantially lower SWB than the general Australian sample. They also reported lower overall life satisfaction and lower perceived control. All this attests to the robust psychometric nature of the PWI as a measurement scale.

These findings from the PWI can be compared with those of Hutton et al. (2013), who also reported lower SWB for people living with HIV. However, the current ESKD sample reported SWB levels 10 points higher than the HIV patients $(M=64.7, S D=19.2$ compared to $M=54.7, S D=21.7)$. Moreover, for the HIV patients, the domain of community connectedness revealed the largest discrepancy, whereas for the current study health satisfaction was the greatest domain source of difference. This seems intuitively reasonable given that HIV is associated with much social stigma, which is likely not an issue for people with ESKD whose condition arguably poses a greater impediment to their capacity to partake in ordinary every-day tasks. These findings highlight the differential impact of various health conditions to SWB, and suggest that targeted interventions addressing the most important domain of SWB for each group may be most efficient.

Finding that the disease-specific measures of HRQOL only weakly predicted general life satisfaction has important implications for data interpretation. Using such scales exclusively would lead to an overestimation of the impact of patients' health status on overall quality of life. This finding is particularly notable since the data were collected while the respondents were on dialysis, and so their health circumstances would have been highly salient. This finding is also consistent with the general understanding that satisfaction with health is not the most important domain contributing to GLS, even for people who are very ill. It is also consistent with the understanding that objective health status is not an adequate measure of perceived quality of life (Cummins 2010a; Okun and George 1984). Thus, although the KD-QOL and DSI scales provide important 
information about the health-related aspects of an individual's quality of life, these measures are not sufficient alone to capture general wellbeing.

The results also reveal that, although SWB was lower for the ESKD sample compared to the general Australian sample, there were no differences between the groups in terms of their self-esteem. This finding attests to the stable, trait-like properties of self-esteem (Trzesniewski et al. 2003) and suggests that self-esteem may be resistant to influence by objective health conditions. It may be inferred that, as a representation of value and worth, self-esteem is deeply ingrained, and not easily threatened by objective circumstances. Thus, although a serious health condition like ESKD may decrease general mood and life satisfaction, a patient is able to separate them self from their condition and/or treatment (Timms et al. 2007), and maintain a sense of worth despite physical limitations.

\section{Limitations}

Though these findings have important implications for understanding the quality of life for people with ESKD, some methodological limitations are noted. Though the study contained a reasonably large sample $(N=172)$, nevertheless it excluded people not fluent in English, who constitute a substantial proportion of the migrant ESKD population. In addition, the comparative Australian group does not represent an ideal control group and may have coincidentally included a number of participants who were also experiencing a chronic health condition. Finally, neither the duration of dialysis nor co-morbidity status were captured in the present study. It is possible that either of these variables could exert a systematic influence on the results that have been reported, and these should be considered in future studies of the wellbeing of this population.

\section{Conclusions}

The results support the use of the Personal Wellbeing Index as a valid and reliable assessment tool to measure the quality of life of people with ESKD. The use of this instrument provides additional clinically-useful information about a person's overall quality of life than can be ascertained using an assessment limited to disease-specific concerns. It is recommended that the PWI be used to complement existing HR-QoL measures to better gauge the general quality of life in clinical populations and inform targeted intervention strategies.

\section{References}

Abdel-Kader, K., Myaskovsky, L., Karpov, I., et al. (2009a). Individual quality of life in chronic kidney disease: influence of age and dialysis modality. Clinical Journal of the American Society of Nephrology, 4, 711-718.

Abdel-Kader, K., Unruh, M. L., \& Weisbord, S. D. (2009b). Symptom burden, depression, and quality of life in chronic and end-stage kidney disease. Clinical Journal of the American Society of Nephrology, 4, $1057-1064$.

Al-Arabi, S. (2006). Quality of life: subjective descriptions of challenges to patients with end stage renal disease. Nephrology Nursing Journal, 33, 285-292. 
Alshraifeen, A., McCreaddie, M., \& Evans, J. M. (2014). Quality of life and well-being of people receiving haemodialysis treatment in Scotland: a cross-sectional survey. International Journal of Nursing Practice, 20, 518-523.

Bennett, P. N., Weinberg, M. K., Bridgman, T., \& Cummins, R. A. (2015). The happiness and subjective wellbeing of people on haemodialysis. Journal of Renal Care, 41, 156-161.

Bonner, A., Caltabiano, M., \& Berlund, L. (2013). Quality of life, fatigue, and activity in Australians with chronic kidney disease: a longitudinal study. Nursing and Health Sciences, 15, 360-367.

Chan, R., Brooks, R., Erlich, J., et al. (2009). The effects of kidney-disease-related loss on long-term dialysis patients' depression and quality of life: positive affect as a mediator. Clinical Journal of the American Society of Nephrology, 4, 160-167.

Corwyn, R. F. (2000). The factor structure of global self-esteem among adolescents and adults. Journal of Research in Personality, 34, 357-379.

Cummins, R. A. (2010a). Fluency disorders and life quality: subjective wellbeing vs. health-related quality of life. Journal of Fluency Disorders, 35, 161-172.

Cummins, R. A. (2010b). Subjective wellbeing, homeostatically protected mood and depression: a synthesis. Journal of Happiness Studies, 11, 1-17.

Cummins, R. A., \& Weinberg, M. K. (2012). Australian unity longitudinal wellbeing study. Deakin University: Australian Centre on Quality of Life, Melbourne, Australia.

Cummins, R. A., \& Wooden, M. (2013). Resilience in times of crisis: the implications of SWB homeostasis and set-points. Journal of Happiness Studies.

Cummins, R. A., Eckersley, R., Pallant, J., van Vugt, J., \& Misajon, R. (2003). Developing a national index of subjective wellbeing: the Australian unity wellbeing index. Social Indicators Research, 62, 159-190.

Cummins, R. A., Woerner, J., Weinberg, M., et al. (2013). Australian unity wellbeing index: - report 29.0 - the wellbeing of Australians - two extra hours, mothers and mothers-in-law. Melbourne: Australian Centre on Quality of Life, School of Psychology, Deakin University.

Diener, E. D., Emmons, R. A., Larsen, R. J., et al. (1985). The satisfaction with life scale. Journal of Personality Assessment, 49, 71-75.

Diener, E., Suh, E. M., \& Lucas, R. E. (1999). Subjective well-being: three decades of progress. Psychological Bulletin, 125, 276-302.

Ekelund, M., \& Andersson, S. I. (2007). Elucidating issues stressful for patients in predialysis and dialysis: from symptom to context. Journal of Health Psychology, 12, 115-126.

Epstein, R. M., Fiscella, K., Lesser, C. S., \& Stange, K. C. (2010). Why the nation needs a policy push on patient-centered health care. Health Affairs, 29(8), 1489-1495.

Hays, R. D., Kallich, J. D., Mapes, D. L., et al. (1994). Development of the Kidney Disease Quality of Life (KDQOLTM) instrument. Quality of Life Research, 3, 329-338.

Hu, L. T., \& Bentler, P. M. (1999). Cutoff criteria for fit indexes in covariance structure analysis: conventional criteria versus new alternatives. Structural Equation Modeling, 6(1), 1-55.

Hutton, V., Misajon, R., \& Collins, F. (2013). Subjective wellbeing and 'felt' stigma when living with HIV. Quality of Life Research, 22, 65-73.

Ibrahim, S., \& El Salamony, O. (2008). Depression, quality of life and malnutrition-inflammation scores in hemodialysis patients. American Journal of Nephrology, 28, 784-791.

Karamanidou, C., Weinman, J., \& Horne, R. (2014). A qualitative study of treatment burden among haemodialysis recipients. Journal of Health Psychology, 19, 556-569.

Lau, A. L. D., Chi, I., Cummins, R. A., et al. (2008). The SARS (Severe Acute Respiratory Syndrome) pandemic in Hong Kong: effects on the subjective wellbeing of elderly and younger people. Aging \& Mental Health, 12, 746-760.

Lovibond, S.H., \& Lovibond, P.F. (1995). Manual for the depression anxiety stress scales. (2nd Ed.). Sydney: Psychology Foundation. ISBN 7334-1423-0.

Marsh, H. W. (1996). Positive and negative global self-esteem: a substantively meaningful distinction or artifactors? Journal of Personality and Social Psychology, 70, 810-819.

Martin, C. R., Thompson, D. R., \& Chan, D. S. (2006). An examination of the psychometric properties of the Rosenberg Self-Esteem Scale (RSES) in Chinese acute coronary syndrome (ACS) patients. Psychology, Health \& Medicine, 11, 507-521.

OECD (2013). OECD guidelines on measuring subjective well-being. OECD Publishing. doi: 10.1787/ 9789264191655-en.

Okun, M. A., \& George, L. K. (1984). Physician- and self-ratings of health, neuroticism and subjective wellbeing among men and women. Personality and Individual Differences, 5, 533-539.

Owens, T. J. (1994). Two dimensions of self-esteem: reciprocal effects of positive self-worth and selfdeprecation on adolescent problems. American Sociological Review, 59, 391-407. 
Pallant, J. (2007). SPSS survival manual: A step by step guide to data analysis using SPSS for windows. Sydney: Allen \& Unwin.

Pearlin, L. I., \& Schooler, C. (1978). The structure of coping. Journal of Health and Social Behavior, 19, 2-21. Rosenberg, M. (1965). Society and the adolescent self-image. New Jersey: Princeton University Press.

Roth, M., Decker, O., Herzberg, P. Y., et al. (2008). Dimensionality and norms of the Rosenberg Self-Esteem Scale in a German general population sample. European Journal of Psychological Assessment, 24, 190-197.

Sheasby, J. E., Barlow, J. H., \& Cullen, L. A. (2000). Psychometric properties of the Rosenberg Self-esteem Scale among people with arthritis. Psychological Reports, 86, 1139-1146.

Sinclair, P., Bennett, P., \& Shandahan, B. (2014). Nursing care of people with kidney disorders. In Medicalsurgical nursing: Critical thinking for person-centered care. Frenchs Forest: Pearson Australia, pp. 887-934.

Son, Y. J., Choi, K. S., Park, Y. R., et al. (2009). Depression, symptoms and the quality of life in patients on hemodialysis for end-stage renal disease. American Journal of Nephrology, 29, 36-42.

Suh, E., Diener, E., \& Fujita, F. (1996). Events and subjective well-being: only recent events matter. Journal of Personality and Social Psychology, 70, 1091-1102.

Tafarodi, R. W., \& Swann, W. B., Jr. (1995). Self-liking and self-competence as dimensions of global selfesteem: initial validation of a measure. Journal of Personality Assessment, 65, 322.

The International Wellbeing Group. (2006). Personal wellbeing index - adult. Melbourne: The Australian Centre on Quality of Life, Deakin University.

Timms, S., King, L., \& Bennett, P. N. (2007). Empowerment for people with end stage renal disease: a literature review. Renal Society of Australia Journal, 3(2), 52-28.

Trzesniewski, K. H., Donnellan, M. B., \& Robins, R. W. (2003). Stability of self-esteem across the lifespan. Journal of Personality and Social Psychology, 84, 205-220.

Weisbord, S. D., Fried, L. F., Arnold, R. M., et al. (2004). Development of a symptom assessment instrument for chronic hemodialysis patients: the dialysis symptom index. Journal of Pain and Symptom Management, 27, 226-240.

WHO. (2013). Joint meeting of experts on targets and indicators for health and well-being in Health 2020. Copenhagen: WHO Regional Office for Europe.

WHO Regional Office for Europe (2012). Measurement of and target-setting for well-being. Second meeting of the expert group, Paris, 25-26 June 2012. Copenhagen: WHO Regional Office for Europe. 